\title{
Enfermedad Trofoblástica (Mola Hidatiforme)
}

\author{
Dr. Alberto Moreno Rojas* \\ "Estudio de 14 casos atendidos en el Hospital General de Neiva"
}

\section{INTRODUCCION}

La enfermedad trofoblástica, nombre que incluye la Mola Hidatiforme y el Cariocarcinoma, representa un problema patológico excepcionalmente complicado; el interés de su estudio ha aumentado en los últimos años debido al mayor conocimiento de las propiedades biológicas y de su importancia diagnóstica $y$ pronóstica. Todavía nos queda mucho por aprender con respecto a estas anormalidades del embarazo pues el cuadro clínico no siempre sigue las reglas convencionales de su tipo histológico (1).

\section{MOLA HIDATIFORME}

Se caracteriza esta forma de la enfermedad trofoblástica por la severa deformación, parcial o total, de las vellosidades coriales, a cuyo nivel ocurren tres fenómenos principales: degeneración hidrópica, desintegración de su vasculatura y proliferación trofoblástica de intensidad variable. Hertig y Mansell creen que

* Médico Ginecoobstetra.

Departamento de Ginecología y Obstetricia Hospital General de Neiva. la Mola es un proceso degenerativo, aunque capaz de transformación neoplásica (2). Baggish en su hipótesis dice que la Mola puede surgir por endo reduplicación del segundo cuerpo polar (3). (4). Carr ha sugerido que los cambios hidrópicos son especialmente comunes después de la suspensión de anticonceptivos orales (5).

Se han emitido otras varias hipótesis etiológicas para explicar la génesis de la Mola: de origen cromosómico, de origen en los grupos sanguíneos, de origen viral, de trastornos de nidación, de desnutrición protéica y también de bajas condiciones socio-económicas (6).

De acuerdo con algunos estudios publicados la mayor parte de los tumores trofoblásticos benignos son de constitución cromosómica $X X(7)$. El número y tamaño de las vesículas molares pueden variar de manera muy apreciable, al igual que su volumen.

\section{OBJETIVOS}

Con esta revisión de casos atendidos en nuestro hospital en el año de 1982. se busca mostrar en una forma más amplia la signología y sintomatología, así 
como la evolución de los pacientes y poder tener una idea más exacta de este tipo de patología ginecoobstétrica. Se comparan los datos obtenidos en esta revisión con los presentados por otros investigadores de diferentes zonas del país, para conocer la magnitud real del problema de salud y tomar medidas pertinentes que ayuden a nuestra comunidad en su bienestar y desarrollo.

\section{MATERIAL Y METODOS}

Se revisaron y analizaron 3.827 casos ginecoobstétricos atendidos en el Hospital General de Neiva en el año de 1982 encontrándose 14 casos de enfermedad trofoblástica (Mola), para una incidencia de $3.6 \times 1.000$. Las preparaciones histológicas realizadas con el material obtenido en los legrados uterinos, de los casos clínicos del presente estudio, fueron revisados de nuevo por el Patólogo del hospital y no hubo variación respecto al diagnóstico anatomopatológico establecido.

\section{RESULTADOS}

Clasificación: La clasificación anatomopatológica adoptada, siguiendo el criterio de Hertig y Mansell (2), mostró los siguientes resultados:

\begin{tabular}{lc}
\hline Mola- & Casos \\
\hline Grado I & 13 \\
Grado II & 1 \\
Grado III & - \\
Cariocarcinoma & - \\
\hline
\end{tabular}

Frecuencia: La frecuencia de la Mola Hidatiforme tiene grandes variaciones de acuerdo con la distribución geográfica y los grupos étnicos, aún dentro del mismo país. El siguiente cuadro muestra la incidencia hospitalaria en Colombia, de acuerdo con los diferentes autores:

\begin{tabular}{llll} 
Autor & Embarazos & Partos & Ciudad \\
\hline Valencia y Col (8) & $1 \times 155$ & & Manizales \\
Acosta-Bendek (9) & $1 \times 311$ & $1 \times 247$ & Barranquilla \\
Vergara Támara (10) & $1 \times 508$ & $1 \times 523$ & Bogotá \\
Duarte Contreras (11) & $1 \times 543$ & $1 \times 324$ & Cúcuta \\
Gómez Palacino (12) & $1 \times 680$ & $1 \times 521-$ & Bogotá \\
Isaza S. (13)' & $1 \times 948$ & & Bogotá \\
Presente estudio & $1 \times 273$ & $1 \times 128$ & Neiva \\
\hline
\end{tabular}

Edad y Paridad:

\begin{tabular}{lclr}
\hline Edad & Casos & Paridad & Casos \\
\hline $15-19$ & 4 & Primigestantes & 7 \\
$20-24$ & 3 & Secundigestantes & 2 \\
$25-29$ & 3 & Tercigestantes & \\
$30-34$ & 1 & Multíparas & 5 \\
$35-39$ & 1 & & \\
$40 y+$ & 2 & & \\
\hline
\end{tabular}

\section{SIGNOS Y SINTOMAS}

Se presentaron con mayor frecuencia después de las 12 semanas de gestación, hecho confirmado por el dato de la fecha de la última menstruación. Hemorragia: fue el signo de mayor frecuencia y se observó en las 14 pacientes $(100 \%)$. Dolor: manifestado por las pacientes como dolor "bajito", fue un síntoma que se encontró en 6 pacientes $(42 \%)$. Vómito: solamente se presentó en 5 ca$\operatorname{sos}(35 \%)$. Cefalea: fue ocasional y de tipo global, solo se presentó en 2 pacientes. Crecimiento uterino: la desproporción entre la amenorrea y la altura uterina se presentó, siendo mayor en 5 casos $(25 \%)$, igual en $4(28 \%)$, y menor en $3(21 \%)$. En 2 historias no estaba consignado este dato. Toxemia: se encontró en una paciente después de eva. cuada la Mola: la evolución clínica de los quistes fue satisfactoria.

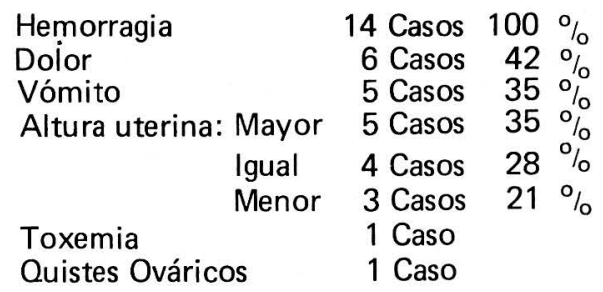




\section{DIAGNOSTICO}

El diagnóstico se basó en la Historia Clínica y el examen de ingreso; en los casos de aborto en el examen del material. Se practicó, como ayuda diagnóstica, ecografía en 5 pacientes. Se tomaron $\mathrm{Rx}$ de tórax a todas las pacientes, en 9 casos un control posterior al tratamiento. No se praticaron amniografías

\section{TRATAMIENTO}

\begin{tabular}{lc}
\hline Método Empleado & No. Casos \\
\hline Inducción & 3 \\
Legrado & 10 \\
Dilatación + legrado & 4 \\
Histerectomía + SOB & 1 \\
Metrotexate & 5 \\
\hline
\end{tabular}

La histerectomía abdominal con SOB fue. practicada después de legrado y perforación uterina. Recibieron Metrotexate 5 pacientes en dosis total de 100 $\mathrm{mg}$ repartidas en dosis fraccionadas durante 5 y hasta 10 días (14) (15).

\section{CONTROLES}

Solamente tres pacientes han regresado a control en forma irregular, del resto de pacientes no se ha tenido información. Previendo esta situación antes de la salida de la paciente del servicio se le comenta en una forma clara cual es su enfermedad, los peligros que pueda tener por falta de control; se le aconseja planificación con DIU y sus controles periódicos en la Institución. No se solicitaron pruebas biológicas ni al ingreso ni durante el tratamiento debido a deficiencias en el laboratorio del Hospital.

\section{COMENTARIOS}

El interés del presente estudio es mostrar la alta incidencia de la enfermedad trofoblástica (Mola) disgnósticada y tratada en el Hospital General de
Neiva, hospital de referencia para todo el Departamento del Huila y regiones vecinas. Podemos observar que la incidencia de la Mola Hidatiforme en el presente estudio es la más alta informada, hasta el momento en el país, dato que se confirma más claramente cuando comparamos con los presentados por los diferentes investigadores.

Los signos y síntomas, en las pacientes que consultaron a nuestro hospital, se acentuaron después de las 12 semanas de gestación, dato tomado de la fecha del primer día de la U.M. informada por la paciente.

El sangrado genital que se observó en todas las pacientes, en un buen porcentaje, empezó tempranamente manifestándose en forma de "manchas oscuras" o "sangrado escaso" antes de la agudización del cuadro cl ínico.

Las pacientes tratadas en nuestra Institución a quienes se les ha ordenado control periódico en la consulta externa del servicio de Ginecología del hospital, ha sido difícil realizarlo por varias razones: falla en el servicio de asistencia social en localizar a las pacientes, el costo de la tarifa hospitalaria en la consulta externa, algunas pacientes viven fuera de la ciudad y otras por cambio de domicilio.

Se les aconseja a las pacientes la planificación con DIU, aunque algunos investigadores como Kistner, comenta que los anticonceptivos orales no afectan el nivel de HCG (16).

Se pone de manifiesto la importancia de elaborar una historia clínica completa a toda paciente en quien se sospeche o diagnostique Mola Hidatiforme; orientar la metodología para el estudio del caso, practicar una buena toma de muestras para enviar a patología y dirigir el tratamiento según la necesidad de la paciente. 


\section{RESUMEN}

Se hizo una revisión de los casos de enfermedad trofoblástica (Mola Hidatiforme) atendidos en el año de 1982 en el Hospital General de Neiva, con el fin de mostrar y comparar la incidencia de dicha patología en nuestro medio.

Se hacen comentarios de acuerdo a los resultados encontrados; se recalca la im- portancia de una buena Historia Clínica y el control de la evolución de las pacientes; se comentan las diferentes fallas hospitalarias y se aconseja buscar mecanismos adecuados para que se pueda hacer un estricto control de estas pacientes sin mayores costos para la usuaria.

\section{TROPHOBLASTIC DISEASE HIDATIFORM MOLE}

\section{Summary}

A careful analysis of all the patients with trophoblastic disease treated during 1982 at Neiva General Hospital was done in order to assess and compare the incidence of such pathology in this milieu.

The article presents comments of the results obtained in the study. Authors

\section{BIBLIOGRAFIA}

1. NOVAK R., Edmun, Mola Hidatiforme y Cariocarcinoma -Patología Ginecológica y Obstétrica. 8a. edición. 33:657, 1982.

2. HERTIG A. Y MANSELL $H$., Atlas of tumors pathology. Section IX, Fascicle 33. Armed Forces Institute of pathology, Washington D.C., 1956.

3. BAGGISH M.S. Gestational trophoblastic Neoplasia. Clin. Obs. Gin. 17:259, 1974.

4. BAGGISH M.S. y Col. Sex Chromatin pattern in Hidatiform Mole, Am. J. Obs. Gin. 102:362, 1968.

5. CARR D.H., Cytogenetics and the pathology of Hydatidiform Degeneration. Obs. Gin. 33:333, 1969. emphasize the importance of a detailed patient record and medical history and of subsequent follow-up to test the evolution of those patients. Hospital deficiencies are pointed out and outhors recommend to look for mechanisms that would facilitate a strict follow-up of these patients at the lowest costs possible for them.
6. REYNOLDS, S.R. Hidatidiform Mole: a Vascular Congenital Anomaly, Obs. Gin. 47:244, 1976.

7. TOMINAGA T. y PAGE E.W. Sex Chromatin of trophoblastic tumors, Am. J. Obs. Gin. 96:305, 1966.

8. VALENCIA F. y Col. Mola Hidatiforme. Rev. Col. Obs. Gin. 15:345, 1964.

9. ACOSTA Bendek, E. Mola Hidatiforme Comentarios. Rev. Col. Obs. Gin. 15:233, 1964.

10. VERGARA T. Roberto y Col. Tumores Trofoblásticos. Rev. Col. Obs. y Gin.18:165, 1967.

11. DUARTE A. y Col. Enfermedad Trofoblástica. Rev. Col. Obs. Gin. 6:585, 1970.

12. GOMEZ Palacino, J.A. y Col. Enferme-dad Trofoblástica Gestacional. Rev. Col. Obs. Gin. 23:469, 1972. 
Vol. XXXIV No. 6

13. ISAZA S.G. Enfermedad Trofoblástica, Rev. Col. Obs. Gin. 30:162, 1979.

14. HERTZ R. y Col. Chemotherapy in women with Trophoblastic Disease. Ann. N. Y. Acad, Sci. 114:881, 1964.
15. JONES W.B. Treatment of Chorionic Tumors. Clin. Osb. Gin. 18:247, 1975.

16. KISTNER R.W. Gynecology. Chicago. Year Book Medical Publishers. Inc. 1964. 